\title{
Solar cycle evolution of dipolar and pseudostreamer belts and their relation to the slow solar wind
}

Article

Published Version

Owens, M. J., Crooker, N.U. and Lockwood, M. (2014) Solar cycle evolution of dipolar and pseudostreamer belts and their relation to the slow solar wind. Journal of Geophysical Research: Space Physics, 119 (1). pp. 36-46. ISSN 2169-9402 doi: https://doi.org/10.1002/2013JA019412 Available at https://centaur.reading.ac.uk/36006/

It is advisable to refer to the publisher's version if you intend to cite from the work. See Guidance on citing.

Published version at: http://dx.doi.org/10.1002/2013JA019412

To link to this article DOI: http://dx.doi.org/10.1002/2013JA019412

Publisher: American Geophysical Union

All outputs in CentAUR are protected by Intellectual Property Rights law, including copyright law. Copyright and IPR is retained by the creators or other copyright holders. Terms and conditions for use of this material are defined in the End User Agreement.

www.reading.ac.uk/centaur 
Central Archive at the University of Reading

Reading's research outputs online 


\section{Journal of Geophysical Research: Space Physics}

\section{RESEARCH ARTICLE}

10.1002/2013JA019412

\section{Special Section:}

The Causes and Consequences of the Extended Solar Minimum between Solar Cycles 23 and 24

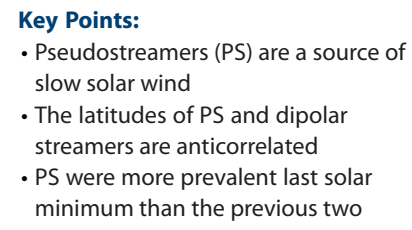

- Pseudostreamers (PS) are a source of slow solar wind

- The latitudes of PS and dipolar streamers are anticorrelated

- PS were more prevalent last solar

minimum than the previous two

\section{Correspondence to:}

M. J. Owens,

m.j.owens@reading.ac.uk

\section{Citation:}

Owens, M. J., N. U. Crooker, and M. Lockwood (2014), Solar cycle evolution of dipolar and pseudostreamer belts and their relation to the slow solar wind, J. Geophys. Res. Space Physics, 119 doi:10.1002/2013JA019412.

Received 4 SEP 2013

Accepted 22 DEC 2013

Accepted article online 27 DEC 2013

\section{Solar cycle evolution of dipolar and pseudostreamer belts and their relation to the slow solar wind}

\author{
M. J. Owens ${ }^{1}$, N. U. Crooker ${ }^{2}$, and M. Lockwood ${ }^{1}$ \\ ${ }^{1}$ Space Environment Physics Group, Department of Meteorology, University of Reading, Reading, UK, ${ }^{2}$ Center for Space \\ Physics, Boston University, Boston, Massachusetts, USA
}

\begin{abstract}
Dipolar streamers are coronal structures formed by open solar flux converging from coronal holes of opposite polarity. Thus, the dipolar streamer belt traces the coronal foot point of the heliospheric current sheet, and it is strongly associated with the origin of slow solar wind. Pseudostreamers, on the other hand, separate converging regions of open solar flux from coronal holes of the same polarity and do not contain current sheets. They have recently received a great deal of interest as a possible additional source of slow solar wind. Here we add to that growing body of work by using the potential-field source-surface model to determine the occurrence and location of dipolar and pseudostreamers over the last three solar cycles. In addition to providing new information about pseudostreamer morphology, the results help explain why the observations taken during the first Ulysses perihelion pass in 1995 showed noncoincidence between dipolar streamer belt and the locus of slowest flow. We find that Carrington rotation averages of the heliographic latitudes of dipolar and pseudostreamer belts are systematically shifted away from the equator, alternately in opposite directions, with a weak solar cycle periodicity, thus keeping slow wind from the web of combined streamer belts approximately symmetric about the equator. The largest separation of dipolar and pseudostreamer belts occurred close to the Ulysses pass, allowing a unique opportunity to see that slow wind from pseudostreamer belts north of the southward displaced dipolar belt was responsible for the noncoincident pattern.
\end{abstract}

\section{Introduction}

The Ulysses mission confirmed the inherently bimodal nature of the solar wind [e.g., Geiss et al., 1995]. At solar minimum, slow, hot solar wind forms a relatively narrow band around the heliographic equator, while the fast, cool wind fills the midlatitude and polar heliosphere, whereas at solar maximum, the slow wind extends to much higher latitudes [McComas et al., 2003]. In this view the slow solar wind is collocated with the dipolar streamer belt, the region where open solar magnetic field lines from opposite polarity polar coronal holes converge to form the heliospheric current sheet. If the dipolar streamer belt is tilted and/or warped with respect to the heliographic equator, fast wind extends to low latitudes and interacts with slow wind to form a heliographic latitudinal band of variability characterized by corotating interaction regions [e.g., Pizzo, 1991]. To first order, then, the maximum latitude of the band of variability follows a solar cycle variation which tracks the latitudinal extent of the heliospheric current sheet [Hoeksema et al., 1982].

The perihelion pass by the Ulysses spacecraft near the end of cycle 22 highlighted a discrepancy in the dipolar streamer model of the slow solar wind. On a synoptic map of solar wind speed generated from both Ulysses and Wind data, Crooker et al. [1997] found that the locus of slowest flow was displaced northward from the dipolar streamer belt, while the streamer belt itself was displaced southward of the heliographic equator. The study reported here shows how this discrepancy arose from the fact that the dipolar streamer belt is not the sole source of slow solar wind.

We now understand that pseudostreamers provide an additional source of slow solar wind [Antiochos et al., 2011; Riley and Luhmann, 2012; Crooker et al., 2012]. Pseudostreamers form at the boundary between coronal magnetic flux from two coronal holes of the same polarity [Eselevich, 1998; Eselevich et al., 1999; Zhao and Webb, 2003; Wang et al., 2007]. Pseudostreamers form arc-like bands, which may not close on themselves, but rather connect to the dipolar streamer belt [Crooker et al., 2012]. We refer to the latitudinal band in which pseudostreamers occur as the "pseudostreamer belt" (as illustrated in Figures 1 and 2), which forms a "web" of slow wind sources [Crooker et al., 2012]. This study shows how the dipolar and pseudostreamer 

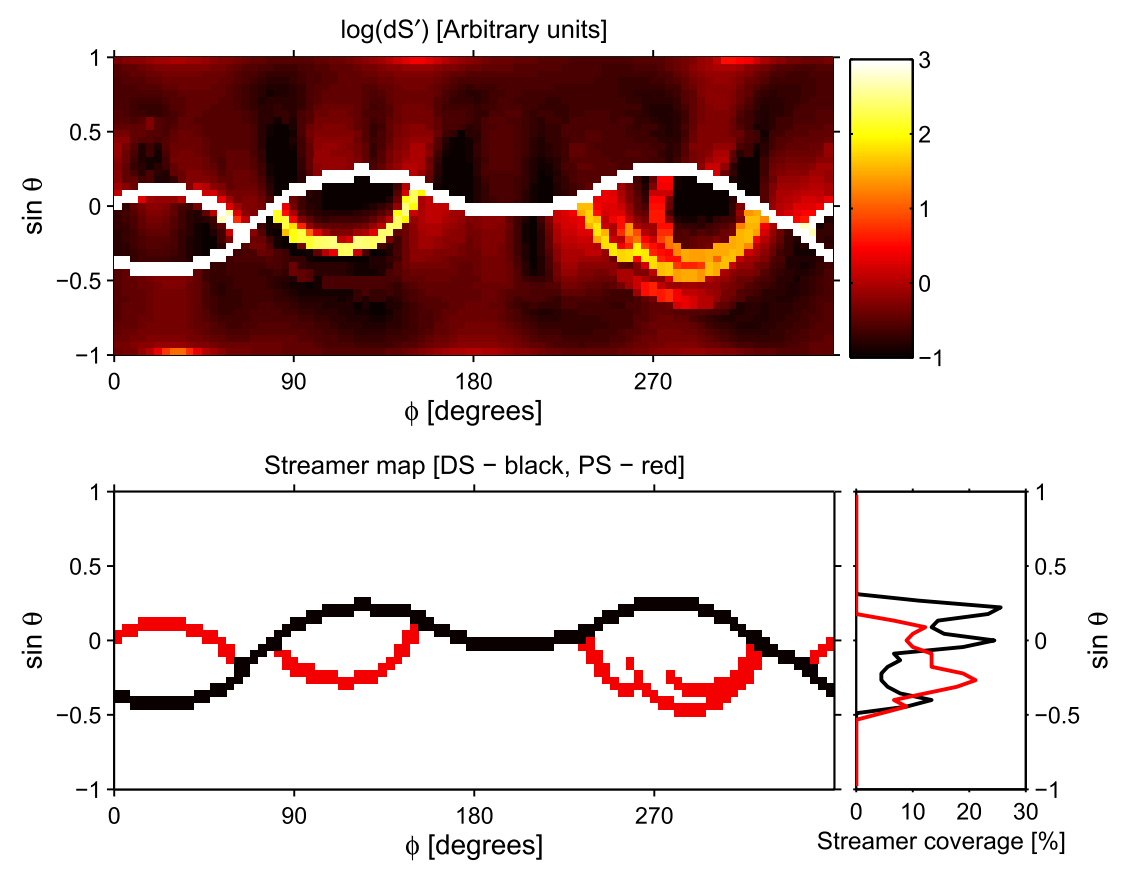

Figure 1. The PFSS solution for Carrington rotation 2056 (May 2007), using a WSO magnetogram. (top) A $\sin \theta-\phi$ map of log(dS') at the source surface. (bottom, left) The streamer map obtained by applying a threshold of $\log \left(\mathrm{dS}^{\prime}\right)>1$. Black (red) lines show the resulting dipolar streamers (pseudostreamers). (bottom, right) Longitudinal averages of dipolar (black) and pseudostreamer (red) occurrence as a function of $\sin \theta$.

components of the web vary over the solar cycle. These results then provide the context for understanding the above mentioned discrepancy in terms of hemispherical asymmetries.

In section 2 we use the potential-field source-surface model [Altschuler and Newkirk, 1969; Schatten et al., 1969] constrained by Wilcox Solar Observatory (WSO) magnetograms to identify the locations of both dipolar and pseudostreamers from 1976 to 2012. In section 4 we compare the occurrence and latitudinal extent of dipolar and pseudostreamer belts over the last three solar cycles, in particular highlighting anomalies in
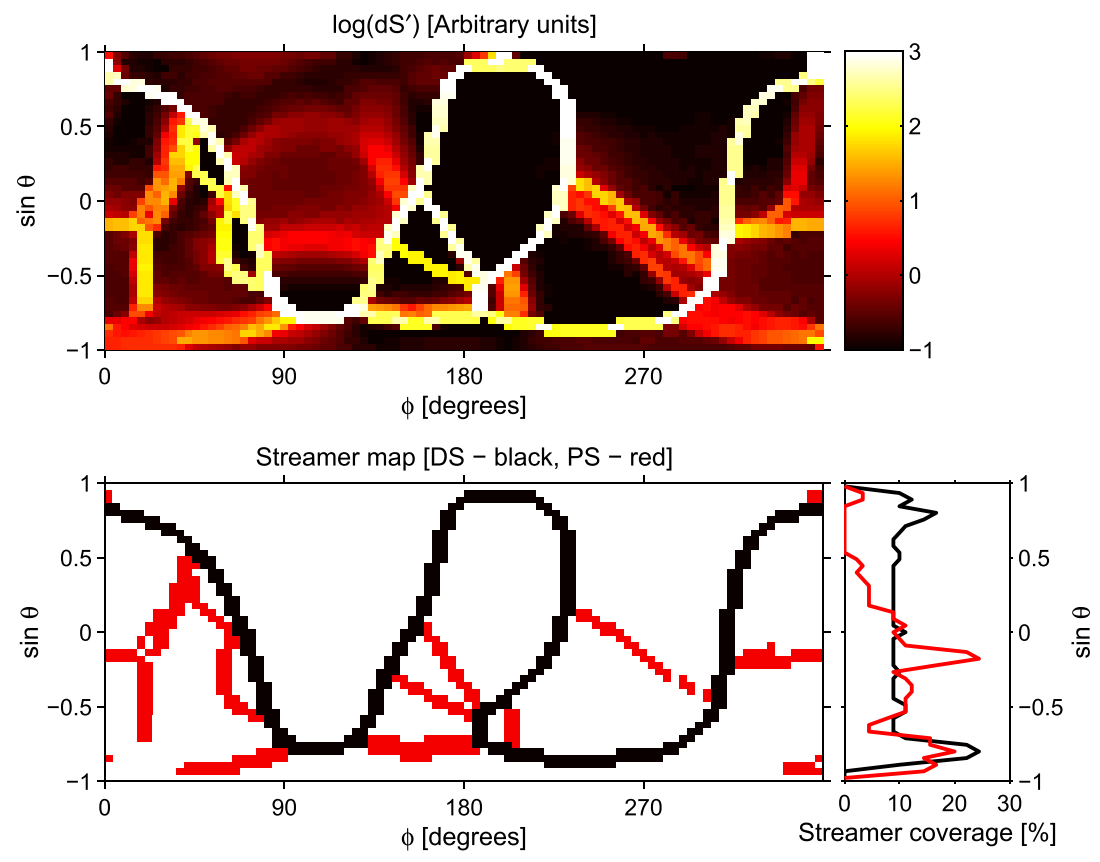

Figure 2. The PFSS solution for Carrington rotation 1955 (approximately spanning October 1999), in the same format as Figure 1. 
the most recent solar minimum between solar cycles 23 and 24. Finally, in section 5 we revisit the two solar minimum Ulysses perihelion passes to determine the relative solar wind contributions from dipolar and pseudostreamers.

\section{Identifying Dipolar and Pseudostreamers}

The potential-field source-surface (PFSS) model is routinely used to infer the magnetic structure of the corona by extrapolating from line-of-sight observations of the photospheric magnetic field [Altschuler and Newkirk, 1969; Schatten et al., 1969]. PFSS solutions are inherently steady state and assume no volumetric currents in the corona, approximating the minimum energy state of the corona for a given photospheric boundary condition. Furthermore, since the acquisition of full longitudinal coverage of the photosphere from ground-based or near-Earth observatories takes approximately 27 days, PFSS solutions are most valid when the corona does not vary significantly over this time (i.e., close to solar minimum). Despite these approximations, the PFSS model has proved to be extremely useful in reconstructing the magnetic field polarity [Arge and Pizzo, 2000] and strength [Wang et al., 2000] at the source surface [see also Mackay et al., 2002, and references therein].

On the basis of approximate empirical relations, the expansion of coronal magnetic flux tubes between the photosphere and the source surface has been used to infer the solar wind speed at the source surface [e.g., Wang and Sheeley, 1990; Arge et al., 2003]. In this model, the fastest solar wind comes from deep within coronal holes, where flux tube expansion is generally limited to that associated with spherical geometry, while slow wind is formed where flux tubes undergo superradial expansion near the edges of coronal holes. Thus, in this approximation, dipolar streamers are the major source of slow solar wind, but pseudostreamers can also contribute if the coronal flux tube expansion is sufficiently high [Wang et al., 2012]. Slow solar wind, however, has been found to be associated with pseudostreamers which do not have large coronal expansion factors [Riley and Luhmann, 2012], and conversely, other pseudostreamers have also been associated with fast solar wind [Panasenco and Velli, 2012].

In this study, dipolar and pseudostreamers are identified in PFSS solutions independent of expansion factors and expected solar wind speed. We use a parameter dS, the distance between photospheric foot points of magnetic flux tubes which are equally spaced on the source surface [Owens et al., 2013]. Since streamers at the source surface are regions where flux tubes of different photospheric origin converge, they are associated with much larger values of dS than the rest of the corona. In many cases, large dS is indeed associated with large coronal flux tube expansion, but there are many instances where it is not [see also Riley and Luhmann, 2012; Panasenco and Velli, 2012]. We note that dS is also closely related to the "squashing factor," $Q$, which quantifies gradients in magnetic field connectivity [e.g., Titov et al., 2011, and references therein]. While $Q$ is a more direct measure of separatrix layers than $\mathrm{dS}$, in practice they identify very similar features. Dipolar streamers are differentiated from pseudostreamers by comparing the polarity of foot points across the narrow peak in dS that identifies a streamer. Different (same) polarities indicate dipolar streamers (pseudostreamers).

Owens et al. [2013] traced magnetic field lines from the source surface to the photosphere on a grid equally spaced in Carrington longitude $(\phi)$ and sine solar latitude $(\sin \theta)$ and identified streamers crossing the ecliptic using a threshold in $\log (\mathrm{dS})$. Because the distance between adjacent points on the source surface grid varies with latitude, both due to the $\sin \theta$ grid spacing and the reduced circumference of the spherical cap, a simple threshold in $\log (\mathrm{dS})$ will preferentially identify more streamers at higher latitudes. Indeed, for some solar minimum PFSS solutions, a log(dS) threshold can misidentify magnetic flux tubes expanding nonradially from within polar coronal holes as pseudostreamers. In this study, we use a modified version of the streamer identification algorithm to identify streamers at all latitudes, not just in the ecliptic plane. Using WSO magnetograms with a potential-source-surface model, we compute $\mathrm{dS}^{\prime}$, such that

$$
\mathrm{dS}^{\prime}=\frac{\mathrm{dS}_{\mathrm{PH}}}{\mathrm{dS}_{\mathrm{SS}}}
$$

where $\mathrm{dS}_{\mathrm{SS}}$ is the distance between points on the source surface and $\mathrm{dS}_{\mathrm{PH}}$ is the distance between their respective points on the photosphere. For each point on the source surface grid, $\mathrm{dS}^{\prime}$ is computed relative to the surrounding eight grid cells in latitude and longitude, and the maximum value is stored. For the highest latitude source surface grid cells, $\mathrm{dS}^{\prime}$ is not calculated. 
In this study, field lines are traced from the source surface using a grid of points equally spaced in $\phi$ and $\sin \theta$. We use 45 grid cells in $\phi$ and 90 grid cells in $\sin \theta$. Increasing or decreasing the spatial resolution at which magnetic field lines are traced changes the magnitude of $\mathrm{dS}^{\prime}$, but the same basic features are present. Most importantly, the fraction and latitudinal extent of streamers are largely unchanged.

Figure 1 (top) shows a $\phi-\sin \theta$ map of $\log \left(\mathrm{dS}^{\prime}\right)$ for Carrington rotation (CR) 2056, which approximately spans May 2007, close to solar minimum at the end of solar cycle 23. The dS' enhancements, shown as bright regions, are confined to the equatorial regions, as expected for the streamer belt close to solar minimum. Figure 1 (bottom, left) shows the result of applying a $\log \left(\mathrm{dS}^{\prime}\right)>1$ threshold. Black points are associated with changing foot point polarity, which classifies them as dipolar streamers, and red points have no polarity change which classifies them as pseudostreamers. The pseudostreamer close to the solar equator at Carrington longitudes $0^{\circ}$ to $50^{\circ}$ has a $\mathrm{dS}^{\prime}$ as large as the dipolar streamer belt. The pseudostreamers at Carrington longitudes $230^{\circ}$ to $320^{\circ}$, however, have lower dS' values, and only parts of the structures seen in Figure 1 (top) meet the $\log \left(\mathrm{dS}^{\prime}\right)>1$ threshold. Thus, while the dipolar streamers form a continuous belt around the Sun collocated with the heliospheric current sheet (HCS), by our selection criteria, pseudostreamers can "fade out" without reaching back to the dipolar streamer belt or other pseudostreamers, even though they must do so topologically [Antiochos et al., 2011]. Figure 1 (bottom, right) shows the percentage of grid points at each latitude which are identified as either dipolar (black) or psuedostreamers (red), equivalent to longitudinally averaged occurrence rate. This again shows that both dipolar and pseudostreamers are confined to the equatorial regions at this phase of the solar cycle, but it also highlights a slight northward shift of the dipolar streamer belt and a slight southward shift of the pseudostreamer belt.

Figure 2 shows Carrington rotation 1955, approximately spanning October 1999, close to the maximum of solar cycle 23. Streamers span a much greater latitudinal extent at this time than during CR2056. The dipolar streamer belt again forms a continuous belt around the Sun, while pseudostreamers branch off both it and each other. For this Carrington rotation, the dipolar streamer belt is approximately symmetric about the helioequator, while pseudostreamer occurrence peaks in the Southern Hemisphere.

Through visual inspection of dS' for all CRs from 1642 to 2120 , the threshold of $\log \left(\mathrm{dS}^{\prime}\right)>1$ was found to be sufficient to identify dipolar streamers along the entirety of the heliospheric current sheet as well as pick out the obvious pseudostreamers. The threshold is arbitrary only to the extent that increasing/decreasing it by an order of magnitude does result in identification of fewer/more pseudostreamers. On the other hand, the threshold is high enough to identify only tight pseudostreamer belts which are only one or two grid cells wide, rather than broad pseudostreamer patches.

\section{Latitudinal Variations Over the Solar Cycle}

Figure 3 (middle and bottom) shows longitudinal averages of the percentage of source surface grid cells associated with dipolar (Figure 3, middle) and pseudostreamers (Figure 3, bottom) as a function of latitude and time. The lower right plots of Figures 2 and 1 are examples of slices through these panels at any one time. Figure 3 (top) gives the sunspot cycles by plotting 27 day means of the international sunspot number. In Figure 3 (middle), the extent of nonzero values (i.e., nonblack regions) follows the maximum latitudinal extension of the HCS (often referred to as the "HCS tilt angle," [Wilcox and Hundhausen, 1983]) and the HCS inclination index [Owens et al., 2011a], as expected. Thus, there is a general "sawtooth" envelope with a sharp rise in the latitudinal extent of dipolar streamers in the rise phase of the solar cycle followed by an extended, approximately linear decline during the declining phase [e.g., Hoeksema et al., 1983]. As was reported by Cliver and Ling [2001], the latitudinal extent of the HCS (and hence dipolar streamers) declines more rapidly in solar cycle (SC) 22 than in SC21 and SC23. It has been suggested that this is related to the odd/even, or peak/trough, variations observed in near-Earth galactic cosmic ray flux [Thomas et al., 2013]. SC21 and SC23, however, do display differences to each other. The SC23 decline is far more protracted than SC21, and the solar minimum at the end of SC23 results in a much broader dipolar streamer (DS) belt than either SC21 or SC22. Indeed, the white/yellow sectors around 1986-1987 and 1995-1996 show that the DS belt was confined close to the solar equator for an extended period, while this situation was only reached for one or two Carrington rotations in 2009. This is discussed further in section 4. There is also some evidence of hemispheric asymmetries in DS occurrence. In particular, the end of SC22 (approximately 1994-1995) shows a shift in the average DS position toward the Southern Hemisphere, in agreement with in situ spacecraft measurements of the HCS at this time [Crooker et al., 1997; Mursula and Hiltula, 2003]. This DS hemispheric 

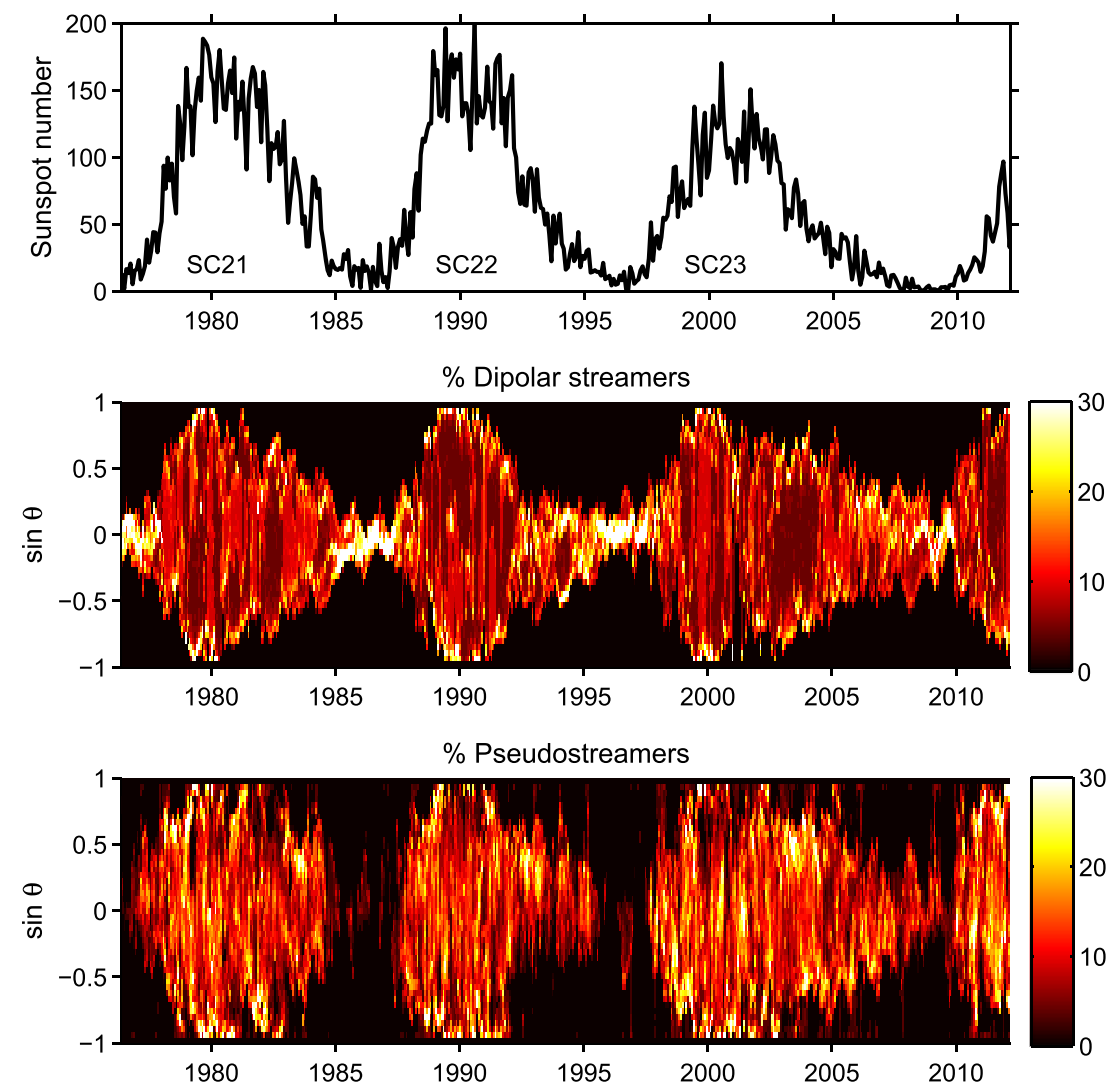

Figure 3. (top) Sunspot number. (middle and bottom) Longitudinal averages of the occurrence rates of dipolar streamers (pseudostreamers) as a function of solar latitude and time.

asymmetry, although less apparent in other solar cycles and at other stages of the solar cycle, is investigated further in section 5 .

The pseudostreamer (PS) occurrence map, in the Figure 3 (bottom), also shows a strong solar cycle variation but with notable differences from the DS map. The PS latitudinal extent increases more rapidly than DS during the rise phase of the solar cycle and decreases more steeply during the declining phase. A striking difference is that pseudostreamers essentially disappear during the minima at the ends of SC21 and SC22 but remain throughout the minimum at the end of SC23. The most prominent hemispheric asymmetry of PS is at the end of SC22, as for DS, but in the opposite sense, with the Northern Hemisphere containing more PS.

\section{Occurrence Rates of Streamers}

Figure 4 shows time series of occurrence of dipolar and pseudostreamers as a percentage of the total source surface (Figure 4, top) and percentage of the ecliptic plane's intersection with the source surface (Figure 4, middle). Thin lines show one Carrington rotation averages produced from PFSS solutions, while thick lines show 1 year averages. The inclination of the ecliptic plane with the helioequator means polar magnetic fields are preferentially observed in the Northern/Southern Hemispheres on a half yearly basis; thus, annual means remove any signal created from this observational effect. As inferred from Figure 3, there is a strong solar cycle variation in both DS and PS occurrence. Given the HCS must always encircle the Sun, the minimum possible DS global occurrence is given by a single continuous DS belt forming a perfect great circle on the source surface, resulting in approximately $4 \%$ global DS coverage at the source surface grid resolution used in this study. This condition is approached at solar minimum, when the DS belt spans the solar equator. Since the ecliptic plane and solar equator are approximately colocated, the ecliptic DS occurrence is peaked at solar minimum, as seen in Figure 3 (middle). Note that the DS belt is not as confined to the ecliptic plane during the minimum at the end of $\mathrm{SC} 23$ as it was at the end of $\mathrm{SC} 21$ and SC22. 


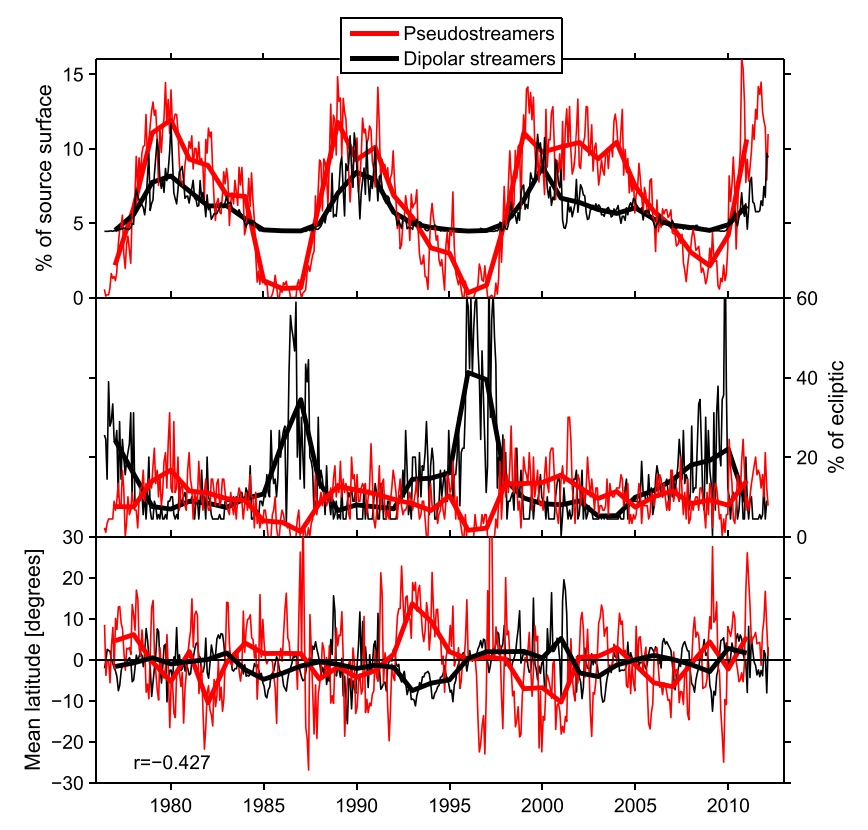

Figure 4. Time series of DS (black) and PS (red) occurrence over the last three solar cycles. Thick (thin) lines show 1 year (Carrington rotation) averages. (top) The global occurrence of DS and PS structures, as a percentage covering of the total source surface. (middle) The percentage of the ecliptic plane at the source surface covered by DS and PS structures. (bottom) The mean latitude of DS and PS occurrence.

Returning to Figure 4 (top), we see that global PS occurrence is comparable to DS occurrence at solar maximum but drops to near zero at solar minimum, resulting in a much higher amplitude solar cycle variation. (We note that using different $\mathrm{dS}^{\prime}$ thresholds changes the magnitude of PS occurrence, but the solar cycle trends are essentially the same). Both global and ecliptic PS occurrence reaches zero at the minima of SC21 and SC22, in 1985-1986 and 1995-1996, but not during the minimum of SC23, in 2008-2009. SC23 is also different from the previous two cycles in that the PS occurrence during the declining phase remains elevated long after the DS occurrence has decreased.

The PS and DS variations can also be considered in terms of solar cycle phase, using solar cycle start/end times of 1976.6, 1986.7, 1996.8, and 2009.2 [Owens et al., 2011b]. (Plots normalized by phase are not shown, but the features are apparent in Figure 4.) Many of the differences between cycles are then removed. All three cycle exhibit a rise in DS occurrence to approximately $8 \%$ within about a quarter of a solar cycle, though SC23 then declines more rapidly in the second quarter of the cycle than in either SC21 or SC22. In the declining phase of the cycle, both SC21 and SC23 retain a greater fraction of DS than SC22, in agreement with the HCS tilt between odd and even cycles [Cliver and Ling, 2001; Thomas et al., 2013]. All three cycles show a rise to a maximum PS occurrence of around $10 \%$ within a quarter of the solar cycle. During the declining phase, PS occurrence in SC23 remains more elevated than in SC21 and SC22. This is the most notable SC23 anomaly which cannot be explained by increased solar cycle length.

\section{Hemispheric Asymmetry and Association of Streamers With Slow Solar Wind}

Hemispheric asymmetries in the time variations of DS and PS locations are apparent in Figure 4 (bottom). There is a clear and persistent anticorrelation between the mean latitude of DS (black) and PS (red) occurrence. When the dipolar streamer belt is displaced northward, the pseudostreamer belts are displaced southward, and vice versa, so that the web of slow solar wind emerging from both PS and DS tends to be centered on the equator. Although the linear correlation coefficient (using Carrington rotation averages) is only -0.43 , it is statistically significant at the $95 \%$ confidence level for the given number of data points. There also appears to be a weak solar cycle periodicity in the mean latitudes, with PS occurrence biased to the Northern (Southern) Hemisphere at solar minimum (maximum).

The largest hemispheric asymmetry occurred during 1992-1995, when the PS belt was shifted more than $10^{\circ}$ northward and the DS belt was centered at approximately $5^{\circ}$ southward. Fortuitously, the Ulysses 


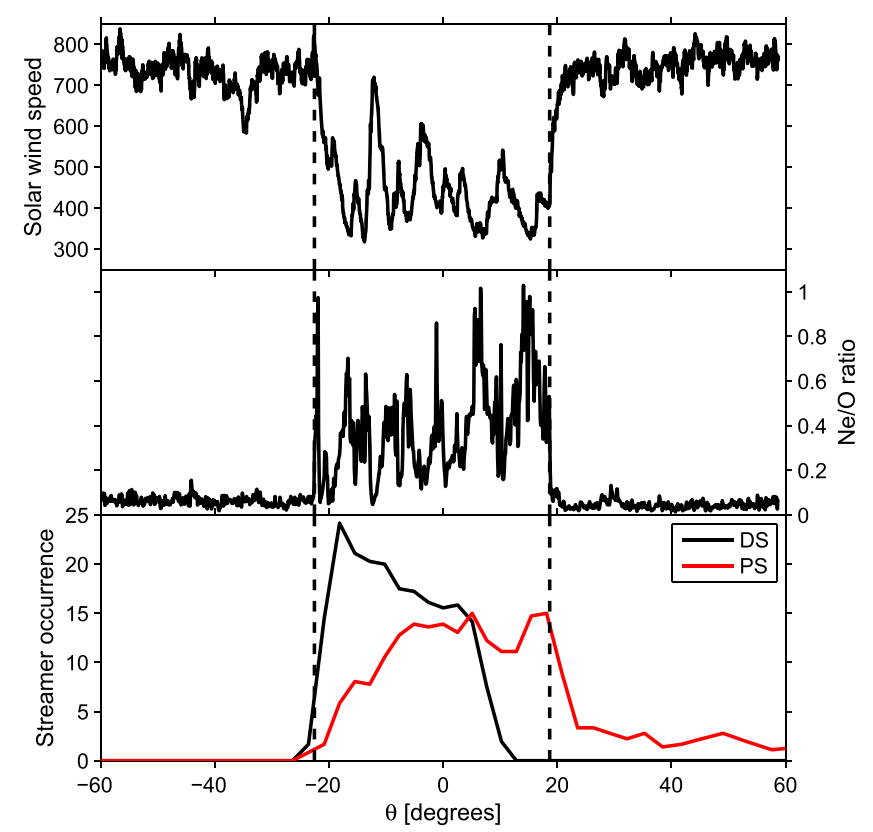

Figure 5. Ulysses in situ observations and PFSS model estimates during the first Ulysses perihelion pass (January-March 1995), as a function of solar latitude. (top) Solar wind speed. (middle) The Ne/O ratio, a proxy for coronal source temperature. A threshold of 0.2 is used to define the band of solar wind variability, shown as the vertical dashed lines. (bottom) The average DS and PS occurrence during the four Carrington rotations in which Ulysses passed from $-40^{\circ}$ to $40^{\circ}$ latitude.

perihelion pass that uncovered the discrepancy in the dipolar streamer model, discussed in section 1 , occurred at the start of 1995. This pass provides a unique opportunity to investigate the solar wind originating from DS and PS regions. We note that because this period is very close to solar minimum, the corona is relatively stable over a number of Carrington rotations, which makes the PFSS solution particularly reliable.

Figure 5 shows a summary of Ulysses in situ solar wind observations and PFSS estimates during the first perihelion pass (taken to be 1995.0 to 1995.3 , the time between which Ulysses traveled from $-40^{\circ}$ to $40^{\circ}$ latitude). Figure 5 (top) shows $1 \mathrm{~h}$ averages of solar wind speed from the Solar Wind Observations Over the Poles of the Sun instrument [Bame et al., 1992], Figure 5, middle shows $3 \mathrm{~h}$ averages of the $\mathrm{Ne} / \mathrm{O}$ abundance ratio from the Solar Wind Ion Composition Spectrometer instrument [Gloeckler et al., 1992], a diagnostic of the temperature of the coronal source of the solar wind. (The more commonly used $\mathrm{O}^{7+} / \mathrm{O}^{6+}$ and $\mathrm{Mg} / \mathrm{O}$ ratio data were both unavailable for this interval.) Throughout this period, there is a strong anticorrelation between coronal temperature and solar wind speed, as expected [Gloeckler et al., 2003]. We take a threshold of $\mathrm{Ne}$ to $\mathrm{O}$ abundances exceeding 0.2 , corresponding to relatively hot coronal source regions, to define the band of variability, shown as the vertical dashed lines, where Ulysses encountered intervals of slow solar wind from the web of dipolar and pseudostreamers.

Figure 5 (bottom) shows the DS and PS occurrence averaged over the four Carrington rotations (CRs 1891 through 1894) of the perihelion pass. Because the corona is so stable at this time, this average is representative of any one of the four rotations. Ulysses' entry into the slow solar wind at approximately $-22^{\circ}$ latitude is coincident with the southernmost extension of the dipolar streamer belt. The DS belt is skewed to the Southern Hemisphere at this time (as seen in Figure 4 (bottom)) and only reaches up to approximately $10^{\circ}$ latitude, while the slow solar wind band extends to nearly $20^{\circ}$. This "extra" slow solar wind appears to be strongly associated with the PS belt, which extends to just over $20^{\circ}$. Thus, the reason for the northward displacement of the slow wind from the dipolar streamer belt found by Crooker et al. [1997] is that the slow wind from pseudostreamers came predominantly from the north side of the dipolar streamer belt. This pattern is clear in the map of $\log \left(\mathrm{dS}^{\prime}\right)$ for $\mathrm{CR} 1893$ shown in Figure 6, where all but one of the pseudostreamer belts lie north of the dipolar belt.

The second Ulysses perihelion pass (not shown) occurred early in 2001, during the solar maximum of SC23. It was not useful for our purposes because the corona at maximum is highly dynamic, and the solar wind 

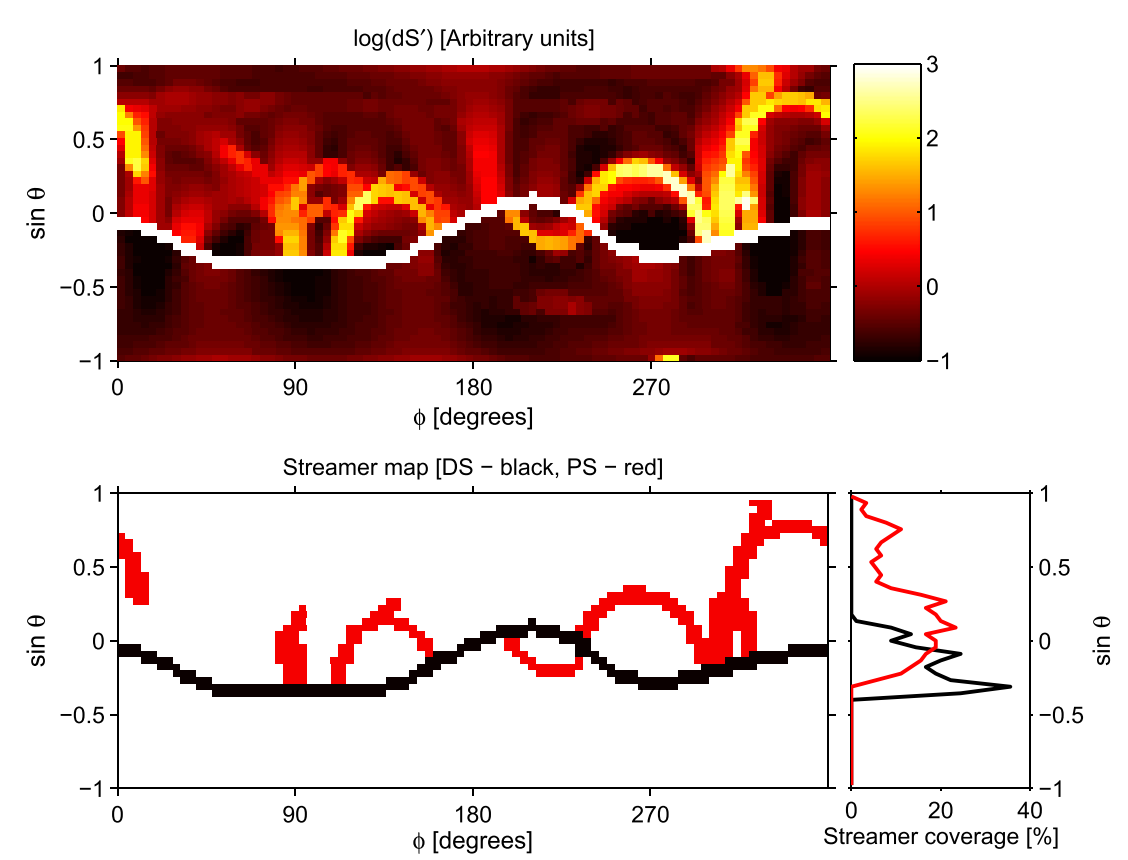

Figure 6. The PFSS solution for Carrington rotation 1893, in the same format as Figure 1.

displays no coherent structure with latitude. PS and DS extend to all latitudes so that their relative solar wind contributions cannot be readily separated.

The third Ulysses perihelion pass occurred during June-September 2007, just prior to solar minimum at the end of SC23. Figure 7 shows a summary of Ulysses and PFSS results for this period. The band of variability is much wider than during the first perihelion pass, and there appear to be short excursions back into slow wind beyond the marked region. The PFSS streamer belts are also wider, with the PS (DS) belt slightly offset to the south (north). Although the belts overlap at most latitudes, the net latitudinal offset is striking because it is opposite to that in Figure 5 . Here the pseudostreamer belts lie predominantly south rather than north of the dipolar belt. This pattern can already be seen in the map of log $\left(\mathrm{dS} \mathrm{S}^{\prime}\right)$ for CR2056

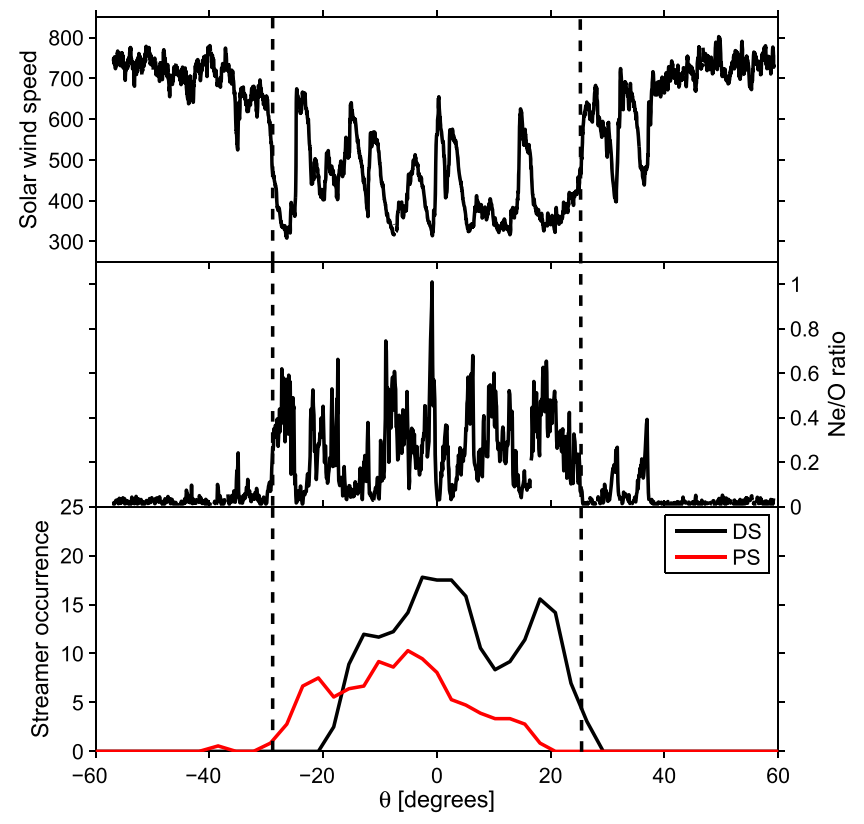

Figure 7. The third Ulysses perihelion pass (June-September 2007), in the same format as Figure 5. 
shown in Figure 1, just prior to the Ulysses pass. Of the three pseudostreamer belts there, two lie south of the dipolar belt, in contrast to the opposite pattern in the map of $\log \left(d S^{\prime}\right)$ for CR1893 shown in Figure 6.

\section{Summary and Discussion}

We have outlined a method for identifying solar wind streamers in potential-field source-surface solutions which is independent empirical relations to solar wind speed. The polarities of the field on the two sides of the streamer readily identify dipolar streamers (DS) and pseudostreamers (PS). Applying this algorithm to Wilcox Solar Observatory magnetograms from 1976 to 2013, we find the following:

1. The latitudinal extent of the DS belt is governed by the heliospheric current sheet "tilt angle" and hence shows an approximately sawtooth variation over the solar cycle. The PS latitudinal extent also shows a solar cycle variation, but with a more rapid growth and decline.

2. Pseudostreamers disappeared during the minima at the end of solar cycles 21 and 22, while dipolar streamers were confined to the solar equator. During the minimum at the end of solar cycle (SC) 23 , however, pseudostreamers persisted and dipolar streamers were less tightly confined to the solar equator.

3. Pseudostreamers were more abundant during the declining phase of SC23 compared to SC21 and SC22. This effect can be largely attributed to the extended length of SC23.

4. The mean latitudes of the PS and DS belts are anticorrelated, with the result that the average streamer belt is approximately symmetric about the solar equator. The PS and DS mean latitudes also display a weak solar cycle variation.

5. The largest hemispheric asymmetry occurred during the end of SC22, when the PS (DS) belt was displaced $10^{\circ}$ northward ( $5^{\circ}$ southward). This pattern was responsible for what was, at the time it was reported by Crooker et al. [1997], a puzzling displacement of the dipolar streamer belt from the locus of slow flow.

6. The latitudinal extent of the domain of slow solar wind in the band of variability observed by Ulysses can only be explained by a combination of dipolar and pseudostreamer contributions.

Implicit in our discussion throughout much of this paper is the assumption that pseudostreamers are a source of slow wind. As mentioned in section 2, this is not always true [e.g., Panasenco and Velli, 2012], but it appears to be true most of the time. Signatures of slow wind from pseudostreamers, first identified by Neugebauer et al. [2004], are commonly found on the slow wind side of stream interfaces at 1 AU [Crooker and McPherron, 2012]. While their speeds are somewhat elevated compared to slow wind from the dipolar streamer belt, their $\mathrm{O}^{7+} / \mathrm{O}^{6+}$ levels meet the criteria for slow wind [Zhao et al., 2009]. Moreover, synoptic maps of solar wind speed predicted by the Wang-Sheeley-Arge model [Arge et al., 2003] show webs of slow wind sources that in most cases, match the source surface pattern of linked dipolar and pseudostreamers [Crooker et al., 2012]

The solar cycle variations in DS and PS occurrence appear to approximately follow the solar quadrupole moment variation inferred by Wang et al. [2002], suggesting they are related to solar magnetic field complexity. Increasing numbers and strength of coronal active regions distort open flux foot points away from the polar coronal holes which dominate at solar minimum, which increases the warped nature of the heliospheric current sheet [e.g., Riley et al., 2002] and hence the total length of the dipolar streamer belt. Similarly, as coronal hole morphology increases in complexity, there is more opportunity for open flux from distant regions of the same coronal hole (or from separate coronal holes of the same polarity) to converge at the source surface, forming pseudostreamers. The rapid formation/disappearance of pseudostreamers over the solar cycle suggests there is a threshold of complexity for PS formation. It is likely that this complexity is governed by the strength of small-scale ephemeral regions relative to that of the low-order magnetic field components so may increase as the dipolar magnetic field weakens.

The declining phase of SC23 and the minimum at the end of SC23 were unusual in a number of ways. Pseudostreamers were more common through the declining phase than during the same phase SC21 and SC22, though this can be at least partly attributed to the extended length of SC23. Increased solar cycle length, however, cannot explain the persistence of pseudostreamers and the increased DS latitudinal range through the minimum at the end of SC23. We speculate that this is due to weaker polar fields and hence a weaker dipolar moment of the global solar magnetic field which allows the higher order moments to dominate [Wang et al., 2009]. The increased PS and reduced DS abundance in the ecliptic plane compared to the minima at the end of SC21 and SC22 may explain the differences in the near-Earth solar wind [Lee et al., 2009; Luhmann et al., 2009; Cliver and Ling, 2011]. Extrapolating these trends to grand solar minima, when the solar 
Acknowledgments

This work was partly facilitated by the ISSI workshop 233, "Long-term reconstructions of solar and solar wind parameters" organized by L. Svalgaard, E. Cliver, J. Beer, and M. Lockwood. We are grateful to T. Hoeksema of Standford University for WSO magnetograms and have benefitted from the availability of Ulysses SWOOPS (PI: D McComas) and SWICS (PI: G. Gloeckler) data. Research for this paper was supported in part (NUC) by the U.S. National Science Foundation under grant AGS-0962645.

Philippa Browning thanks Charles Smith and an anonymous reviewer for their assistance in evaluating this paper. magnetic field strength is greatly reduced [e.g., Owens et al., 2012], suggests an even larger fraction of the heliosphere would be filled with slow solar wind.

Perhaps the most surprising finding of this study is the systematic way in which pseudostreamer belts seem to balance out the spatial distribution of slow wind sources in the course of a solar cycle. When the dipolar streamer belt is displaced southward (northward), the pseudostreamer belts extend northward (southward). Examples of opposite displacement patterns have been presented with data from the first and third Ulysses perihelion passes. They demonstrate that both dipolar and pseudostreamer belts are required to account for the full width of the band of variability created by slow wind sources. This may result in symmetric solar momentum fluxes from the north and south solar hemispheres, even when magnetic asymmetries are present.

\section{References}

Altschuler, M. A., and G. Newkirk Jr. (1969), Magnetic fields and the structure of the solar corona, Sol. Phys., 9, 131-149.

Antiochos, S. K., Z. Mikić, V. S. Titov, R. Lionello, and J. A. Linker (2011), A model for the sources of the slow solar wind, Astrophys. J., 731, 112, doi:10.1088/0004-637X/731/2/112.

Arge, C. N., and V. J. Pizzo (2000), Improvement in the prediction of solar wind conditions using near-real time solar magnetic field updates, J. Geophys. Res., 105, 10,465-10,479.

Arge, C. N., D. Odstrcil, V. J. Pizzo, and L. R. Mayer (2003), Improved method for specifying solar wind speed near the Sun, Proceedings of the Tenth International Solar Wind Conference, vol. 679, pp. 190-193, Pisa, Italy.

Bame, S. J., D. J. McComas, B. L. Barraclough, J. L. Phillips, K. J. Sofaly, J. C. Chavez, B. E. Goldstein, and R. K. Sakurai (1992), The Ulysses solar wind plasma experiment, Astron. Astrophys. Suppl. Ser., 92, 237-265.

Cliver, E. W., and A. G. Ling (2001), 22 Year patterns in the relationship of sunspot number and tilt angle to cosmic-ray intensity, Astrophys. J. Lett., 551, L189-L192, doi:10.1086/320022.

Cliver, E. W., and A. G. Ling (2011), The floor in the solar wind magnetic field revisited, Sol. Phys., 274, 285-301, doi:10.1007/s11207-010-9657-6.

Crooker, N. U., and R. L. McPherron (2012), Coincidence of composition and speed boundaries of the slow solar wind, J. Geophys. Res., 117, A09104, doi:10.1029/2012JA017837.

Crooker, N. U., A. J. Lazarus, J. L. Phillips, J. T. Steinberg, A. Szabo, R. P. Lepping, and E. J. Smith (1997), Coronal streamer belt asymmetries and seasonal solar wind variations deduced from Wind and Ulysses data, J. Geophys. Res., 102, 4673-4680, doi:10.1029/96JA03681.

Crooker, N. U., S. K. Antiochos, X. Zhao, and M. Neugebauer (2012), Global network of slow solar wind, J. Geophys. Res., 117, A04104, doi:10.1029/2011JA017236.

Eselevich, V. G. (1998), On the structure of coronal streamer belts, J. Geophys. Res., 103, 2021-2027, doi:10.1029/97JA02365.

Eselevich, V. G., V. G. Fainshtein, and G. V. Rudenko (1999), Study of the structure of streamer belts and chains in the solar corona, Sol. Phys., 188, 277-297.

Geiss, J., G. Gloeckler, and R. von Steiger (1995), Origin of the solar wind from composition data, Space Sci. Rev., 72, 49-60, doi:10.1007/BF00768753

Gloeckler, G., T. H. Zurbuchen, and J. Geiss (2003), Implications of the observed anticorrelation between solar wind speed and coronal electron temperature, J. Geophys. Res., 108(A4), 1158, doi:10.1029/2002JA009286.

Gloeckler, G., et al. (1992), The solar wind ion composition spectrometer, Astron. Astrophys. Suppl. Ser., 92, 267-289.

Hoeksema, J. T., J. M. Wilcox, and P. H. Scherrer (1982), Structure of the heliospheric current sheet in the early portion of sunspot cycle 21, J. Geophys. Res., 87(A12), 10,331-10,338.

Hoeksema, J. T., J. M. Wilcox, and P. H. Scherrer (1983), The structure of the heliospheric current sheet: 1978-1982, J. Geophys. Res., 88(A12), 9910-9918.

Lee, C. O., J. G. Luhmann, X. P. Zhao, Y. Liu, P. Riley, C. N. Arge, C. T. Russell, and I. de Pater (2009), Effects of the weak polar fields of solar cycle 23: Investigation using OMNI for the STEREO mission period, Sol. Phys., 256, 345-363, doi:10.1007/s11207-009-9345-6.

Luhmann, J. G., C. O. Lee, Y. Li, C. N. Arge, A. B. Galvin, K. Simunac, C. T. Russell, R. A. Howard, and G. Petrie (2009), Solar wind sources in the late declining phase of cycle 23: Effects of the weak solar polar field on high speed streams, Sol. Phys., 256, 285-305, doi:10.1007/s11207-009-9354-5.

Mackay, D., E. Priest, and M. Lockwood (2002), The evolution of the Sun's open magnetic flux-II. Full solar cycle simulations, Sol. Phys., 209, 287-309.

McComas, D. J., H. A. Elliott, N. A. Schwadron, J. T. Gosling, R. M. Skoug, and B. E. Goldstein (2003), The three-dimensional solar wind around solar maximum, Geophys. Res. Lett., 30(10), 1517, doi:10.1029/2003GL017136.

Mursula, K., and T. Hiltula (2003), Bashful ballerina: Southward shifted heliospheric current sheet, Geophys. Res. Lett., 30(22), 2135, doi:10.1029/2003GL018201.

Neugebauer, M., P. C. Liewer, B. E. Goldstein, X. Zhou, and J. T. Steinberg (2004), Solar wind stream interaction regions without sector boundaries, J. Geophys. Res., 109(A10), 2156-2202, doi:10.1029/2004JA010456.

Owens, M. J., N. U. Crooker, and M. Lockwood (2011a), How is open solar magnetic flux lost over the solar cycle?, J. Geophys. Res., 116, A04111, doi:10.1029/2010JA016039.

Owens, M. J., M. Lockwood, C. J. Davis, and L. Barnard (2011b), Solar cycle 24: Implications for energetic particles and long-term space climate change, Geophys. Res. Lett., 38, L19106, doi:10.1029/2011GL049328.

Owens, M. J., I. Usoskin, and M. Lockwood (2012), Heliospheric modulation of galactic cosmic rays during grand solar minima: Past and future variations, Geophys. Res. Lett., 39, L19102, doi:10.1029/2012GL053151.

Owens, M. J., N. U. Crooker, and M. Lockwood (2013), Solar origin of heliospheric magnetic field inversions: Evidence for coronal loop opening within pseudostreamers, J. Geophys. Res. Space Physics, 118, 1868-1879, doi:10.1002/jgra.50259.

Panasenco, O., and M. Velli (2012), Coronal pseudostreamers: Source of fast or slow solar wind? ArXiv e-prints.

Pizzo, V. J. (1991), The evolution of corotating stream fronts near the ecliptic plane in the inner solar system. 2. Three-dimensional tilted-dipole fronts, J. Geophys. Res., 96, 5405-5420, doi:10.1029/91JA00155. 
Riley, P., and J. G. Luhmann (2012), Interplanetary signatures of unipolar streamers and the origin of the slow solar wind, Sol. Phys., 277, 355-373, doi:10.1007/s11207-011-9909-0.

Riley, P., J. A. Linker, and Z. Mikić (2002), Modeling the heliospheric current sheet: Solar cycle variations, J. Geophys. Res., 107(A7), 1136, doi:10.1029/2001JA000299.

Schatten, K. H., J. M. Wilcox, and N. F. Ness (1969), A model of interplanetary and coronal magnetic fields, Sol. Phys., 9, $442-455$.

Thomas, S. R., M. J. Owens, and M. Lockwood (2013), The 22-year hale cycle in cosmic ray flux-Evidence for direct heliospheric modulation, Sol. Phys., 289(1), 407-421, doi:10.1007/s11207-013-0341-5.

Titov, V. S., Z. Mikić, J. A. Linker, R. Lionello, and S. K. Antiochos (2011), Magnetic topology of coronal hole linkages, Astrophys. J., 731, 111, doi:10.1088/0004-637X/731/2/111

Wang, Y.-M., and N. R. Sheeley Jr. (1990), Solar wind speed and coronal flux-tube expansion, Astrophys. J., 355, 726.

Wang, Y.-M., N. R. Sheeley Jr., and J. Lean (2000), Understanding the evolution of the Sun's open magnetic flux, Geophys. Res. Lett., 27, $621-624$

Wang, Y.-M., N. R. Sheeley Jr., and M. D. Andrews (2002), Polarity reversal of the solar magnetic field during cycle 23, J. Geophys. Res., 107(A12), 1465, doi:10.1029/2002JA009463.

Wang, Y.-M., N. R. Sheeley Jr., and N. B. Rich (2007), Coronal pseudostreamers, Astrophys. J., 658, 1340-1348, doi:10.1086/511416.

Wang, Y.-M., E. Robbrecht, and N. R. Sheeley Jr. (2009), On the weakening of the polar magnetic fields during solar cycle 23, Astrophys. J., 707, 1372-1386, doi:10.1088/0004-637X/707/2/1372.

Wang, Y.-M., R. Grappin, E. Robbrecht, and N. R. Sheeley Jr. (2012), On the nature of the solar wind from coronal pseudostreamers, Astrophys. J., 749, 182, doi:10.1088/0004-637X/749/2/182.

Wilcox, J. M., and A. J. Hundhausen (1983), Comparison of heliospheric current sheet structure obtained from potential magnetic field computations and from observed polarization coronal brightness, J. Geophys. Res., 88, 8095-8096, doi:10.1029/JA088iA10p08095.

Zhao, L., T. H. Zurbuchen, and L. A. Fisk (2009), Global distribution of the solar wind during solar cycle 23: ACE observations, Geophys. Res. Lett., 36, L14104, doi:10.1029/2009GL039181.

Zhao, X. P., and D. F. Webb (2003), Source regions and storm effectiveness of frontside full halo coronal mass ejections, J. Geophys. Res., 108(A6), 1234, doi:10.1029/2002JA009606. 